\title{
Br treated graphite nanoplatelets for improved electrical conductivity of polymer
}

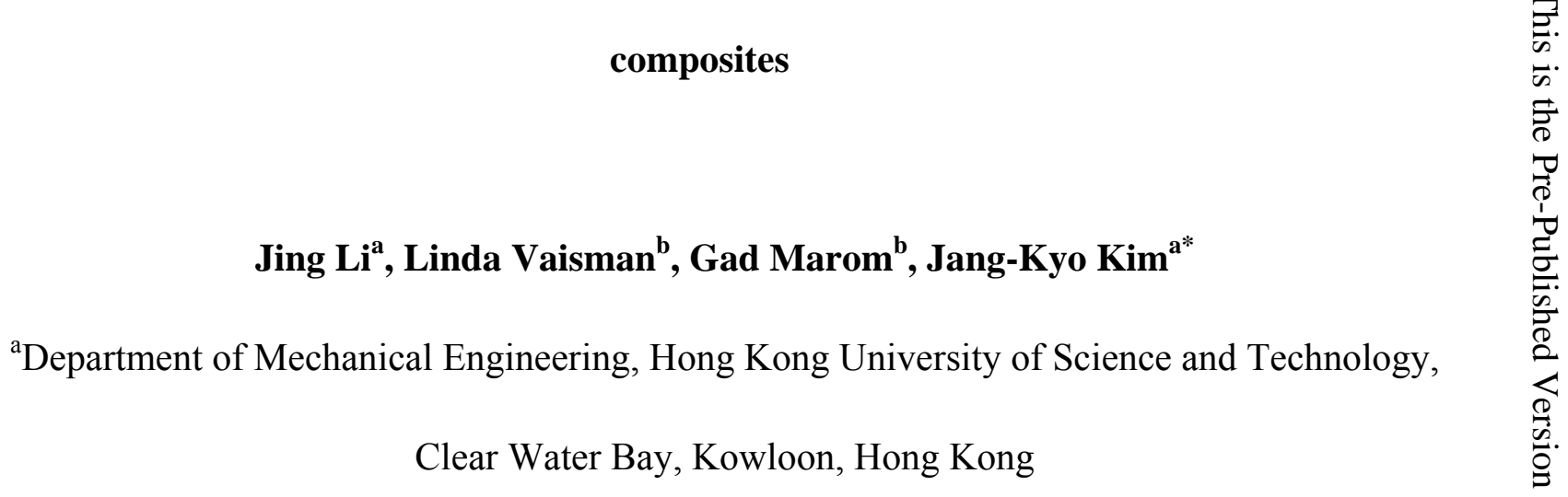

${ }^{\mathrm{b}}$ Casali Institute of Applied Chemistry, The Hebrew University of Jerusalem, Jerusalem, Israel

\section{Abstract}

The graphite nanoplatelets (GNP) were treated by vapor-phase bromination. The increase in weight and atomic concentration of $\mathrm{Br}$ indicated the bromine uptake. The intercalation of $\mathrm{Br}$ between graphene layers of GNP was confirmed by the X-ray diffraction result, showing an increase in the interlayer spacing from $3.342 \AA$ to $3.361 \AA$. Two types of bonds between $\mathrm{C}$ and $\mathrm{Br}$ were introduced simultaneously, ionic and covalent bonds, both of them increased with bromination duration. The fraction of ionic bond reached the highest value by $3 \mathrm{~h} \mathrm{Br}$ exposure, which corresponded to the highest electrical conductivity of GNP. Although the bromination treatment did not change the percolation threshold of composites, it increased the absolute value of electrical conductivity of composites when the filler content was higher than the percolation threshold.

Keywords: Intercalation, Exfoliated graphite, Electrical properties.

*Corresponding author. Fax: 852-23581543. E-mail address: mejkkim@ust.hk (J.K. Kim) 


\section{Introduction}

Graphite nanoplatelet (GNP) is a nanometer-scale conductive filler produced by exfoliating natural flake graphite, and has attracted significant attention as the low cost and lightweight alternative to metal- and carbon-based electrically conducting reinforcements for conducting polymer composites. GNPs are produced from graphite flakes intercalated with high concentrated acids, which can be expanded up to a few hundreds of times along its c-axis at a high temperature. The exfoliation of expanded graphite results in separation of the graphite sheets into nanoplatelets with a very high aspect ratio $[1,2]$. The high aspect ratio and the large surface area of GNPs are responsible for the much lower percolation threshold and better electrical conductivity of conducting polymer composites than micrometer-scale conventional reinforcements. [1,3]

Conducting polymer composites have many potential applications in electromagnetic interference shielding for electronic devices and electrostatic dissipation, where high electrical conductivity of composites materials is the most critical requirement. Therefore, significant efforts have been directed towards improving the electrical conductivity of conducting composites. It has been reported [4,5] that intercalation of guest atoms between the graphene planes forming ionic bonds (charge transfer) can enhance the electrical conductivity of graphitic material by adding electrons to its conduction band or holes to the valence band. Meanwhile, the formation of covalent bonds between the guest atoms and graphite can depress the carrier concentration and displace some of the $\mathrm{C}$ atoms slightly away from the highly planar structure of pristine graphite, which in turn decreases the electrical conductivity.

Amongst many intercalation species, some compounds such as $\mathrm{AsF}_{5}$ would most 
probably generate higher conductivity improvement than bromine [4]. The reasons that we chose bromine as the model compound has three folders: 1) bromine is less toxic and easier to intercalate; 2) brominated carbon fibres showed long term stability under ambient condition [4] and a high thermal stability below $150{ }^{\circ} \mathrm{C}[4,5]$; 3) bromine has intermediate chemical activities in host-guest interactions, compared to Iodine which allows a weak host-guest reactivity, thus introducing only weak charge transfer, and fluorine, which is so strong that covalent bonding can be easily formed with host materials.

Bromine intercalation has been successfully used to increase the electrical conductivity of carbon fibres [4 6]. The charge transfer was the main bonding type between $\mathrm{Br}$ and $\mathrm{C}$, and covalent bonding was introduced only after prolonged reactions [5]. A higher level of graphitization of the host fiber induced better intercalation and higher electrical conductivity of doped carbon fibres $[4,6]$. Vapor-phase bromination treatment has also been applied to nanometer-scale graphite, such as single wall carbon nanotubes [7] and onion-like nanographite particles [8]. A remarkable 30 time increase in electrical conductivity was reported [7] for single wall carbon nanotubes through the formation of charge transfer complexes. Bromine intercalation showed similar effects on the electronic properties of onion-like nanographite [8].

This paper is part of a larger project on fabrication, properties characterization and applications of conducting GNP composites. In our previous studies [2,9], the $\mathrm{UV} / \mathrm{O}_{3}$ treatment technique was successfully applied to improve the electrical conductivity as well as the thermo-mechanical and mechanical properties of GNP/epoxy composites. Here, GNPs were treated with bromine to improve the electrical properties of conducting polymer composites made therefrom. With much larger density of open edges than the other carbon 
materials mentioned above, GNPs would show significant synergy in improving the electrical conductivity after the treatment. X-ray photoelectron spectroscopy (XPS), X-ray diffraction (XRD) analysis and Time-of-Flight Secondary Ion Mass Spectrometry (ToF-SIMS) were employed to evaluate the intercalation states of $\mathrm{Br}$ atoms and quantitatively analyze the bonding types between the $\mathrm{C}$ and $\mathrm{Br}$ atoms.

\section{Experimental}

\subsection{Sample preparation}

GNPs were prepared according to the method described in our previous studies $[2,9]$. Graphite intercalation compound (GIC) containing $2.8 \mathrm{wt} \%$ of sulfur as intercalant (supplied by Asbury Graphite Mills, USA) was put into an oven that was maintained at $1050{ }^{\circ} \mathrm{C}$ and taken out after $30 \mathrm{~s}$ to produce expanded graphite. Upon rapid heating the GIC was expanded explosively several hundred times along the thickness direction due to the evaporation of the intercalant and the thermal shock. The expanded graphite was immersed in acetone and ultrasonicated at 70 watts and $42 \mathrm{kHz}$ for $8 \mathrm{~h}$ to obtain exfoliated GNPs. The thickness and diameter of GNPs were estimated to be $4.5 \mathrm{~nm}$ and $46 \mu \mathrm{m}$ on average [2].

GNPs were exposed to $\mathrm{Br}_{2}$ vapor of the vapor pressure of liquid $\mathrm{Br}_{2}$ at room temperature in a closed desiccator for varying durations. The brominated GNPs were transferred to the fume hood and ventilated to allow desorption of physically absorbed bromine until a constant weight was reached. It is the residual bromine intercalation compound that has technological significance because of its stability in ambient air. The weights of GNPs before and after the treatment were recorded in two sets of identical experiments.

Brominated GNPs were mixed with epoxy (Epon 828, supplied by Shell Chemical) using 
a high-shear mixer for $30 \mathrm{~min}$ at $3000 \mathrm{rpm}$, followed by ultrasonication at 70 watts $42 \mathrm{kHz}$ for $30 \mathrm{~min}$ at $80{ }^{\circ} \mathrm{C}$. The mixture was then outgassed at $80{ }^{\circ} \mathrm{C}$ for $2 \mathrm{~h}$ in a vacuum oven. Curing agent, 1,3-Phenylenediamine (mPDA, supplied by Sigma-Aldrich), was added into the mixture in the ratio of $14.5 / 100$ by weight. Composite plates were cast using an aluminum mould with a cavity of $3 \mathrm{~mm}$ in thickness, which was cured at $80{ }^{\circ} \mathrm{C}$ for $2 \mathrm{~h}$, followed by a post cure at $150{ }^{\circ} \mathrm{C}$ for $3 \mathrm{~h}$. Figure 1 shows the above described process flow of GNPs and the corresponding composites.

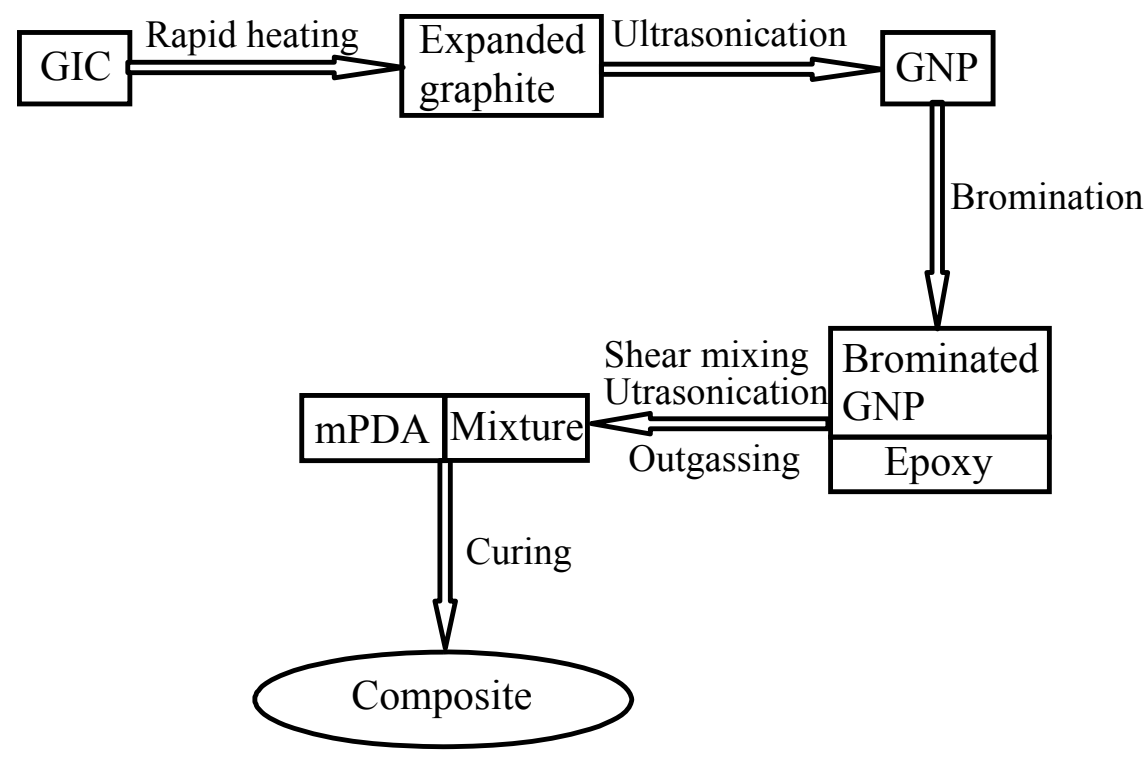

Fig. 1. Process flow of GNPs and the corresponding composites.

\subsection{Characterizations}

The inter-graphene d-spacing of GNPs was determined using a powder X-ray diffractometer (XRD, Philips PW1830). XRD patterns were obtained using a $\mathrm{Cu}-\mathrm{K} \alpha$ radiation $(\lambda=0.154 \mathrm{~nm})$ with a step size of $0.05^{\circ}$ and at a scanning rate of $2.0 \%$ min, working at $45 \mathrm{kV} 40 \mathrm{~mA}$. X-ray photoelectron spectroscopy (XPS, PHI 5600), equipped with a monochromatic Al-Ka X-ray source operated in a residual vacuum of $5 \times 10^{-9}$ Torr, was employed to detect the changes in 
elemental compositions of GNPs after the bromination treatment. The emission angle of the photoelectron, the angle between the sample surface and the axis of the analyzer, was $45^{\circ}$, corresponding to a sampling depth of approximately $4.7 \mathrm{~nm}$ [10]. The pass energy was $187.85 \mathrm{eV}$ for survey scans and $23.5 \mathrm{eV}$ for high-resolution scans. The high-resolution spectra were fitted with multitak software (provided by Physical Electronics). The bonding types between the $\mathrm{C}$ and $\mathrm{Br}$ atoms were analyzed using the time-of-flight secondary ion mass spectrometry (ToF-SIMS, PHI 7200). The primary $\mathrm{Cs}^{+}$ion source was operated at $8 \mathrm{kV}$ and the scanning area was $200 \mu \mathrm{m}$ square. Secondary ions in the mass range from 0 to $600 \mathrm{amu}$ were collected. The XPS and Tof-SIMS results presented below are the mean values and the corresponding standard deviations of three independent measurements obtained for a given set of conditions.

The bulk electrical conductivities of GNPs and GNP/epoxy composites were measured at room temperature using a resistivity/Hall measurement system (Bio-Rad HL5500PC) based on the four probe van der Pauw method. The DC current source of $1 \mathrm{~mA}$ was applied for measuring the conductivity of GNP and $0.1 \mu \mathrm{A}$ for measuring the conductivity of GNP/epoxy composites. GNPs were compacted into tablets of $5 \mathrm{~mm}$ in diameter and $0.5 \mathrm{~mm}$ in thickness using a 10 ton hydraulic press. Composite plates were cut and polished into specimens of $10 \mathrm{~mm}$ square $\mathrm{x} 1 \mathrm{~mm}$ in thickness. As the highest limit of the electrical resistivity measured using the current resistivity/Hall measurement system was $10^{8} \Omega \mathrm{cm}$, the electrical resistivity of composites higher than this limit was measured using a programmable curve tracer (Sony Tektronix 370A) with the lowest DC current source of 1 nA. The initial linear part of I-V curve was used to calculate the electrical resistivity. Silver paste was applied onto the composite specimens to ensure low contact resistance with the 
measuring probes.

Three-point flexure test was performed to measure the mechanical properties of GNP/epoxy composites according to the specification, ASTM standard D790-96. The moulded composite plates were cut into $12.7 \mathrm{~mm}$ wide $\times 70 \mathrm{~mm}$ long x $3 \mathrm{~mm}$ thick samples, which were subjected to bending with a support span of $50 \mathrm{~mm}$ at a constant cross-head speed of $1.3 \mathrm{~mm} / \mathrm{min}$ on a universal testing machine. Five specimens were tested for each set of conditions.

\section{Results and discussion}

\subsection{Bromine uptake and intercalation}

The uptake of bromine by GNP upon its exposure to molecular $\mathrm{Br}$ (generally termedbromination) is expected to become evident by weight increase, which occurs by two parallel routes forming either intercalation complexes or covalent $\mathrm{C}$ - $\mathrm{Br}$ groups. Because only the first is expected to affect the $\pi$ electron interaction between the bromine and GNP, it is important that in addition to the overall uptake the proportion of the two compounds be determined. Figure 2 shows the changes in weight and $\mathrm{Br}$ atomic concentration determined by XPS as a result of bromination. Evidently, the weight increased rapidly with bromination duration and saturated after about $10 \mathrm{~h}$, and then stabilized or decreased slightly. This phenomenon can be explained by the dynamic process of absorption and desorption of bromine atoms into graphite. For treatments below $10 \mathrm{~h}$, bromine was absorbed rapidly because of the ample availability of doping/reactive sites in the GNPs. Upon filling of these sites, further treatment did not induce additional absorption, rather, slight desorption of bromine took place, which can be attributed perhaps to gradual changes in the nature and balance of the $\mathrm{C}$ - $\mathrm{Br}$ bond types 
with bromination duration.

Another evidence for bromine uptake was the increase of $\mathrm{Br}$ atomic concentration with bromination duration determined by XPS, as shown in Figure 2. Although the point of maximum $\mathrm{Br}$ concentration was obtained for the $72 \mathrm{~h}$ treatment, which is slightly different from the result of the weight measurement, the two trends are virtually identical. Noting that the weigh of GNPs was measure in ambient condition, but the XPS experiments were carried out in a residual vacuum of $5 \times 10^{-9}$ Torr, the differences probably arose from the desorption of $\mathrm{Br}$ atoms during the evacuation for the XPS experiment. The XPS results can be taken to express the bulk rather than the surface chemical composition of GNP, because the sampling depth of XPS is up to approximately $4.7 \mathrm{~nm}$, while the thickness of GNP is about $4.5 \mathrm{~nm}$.

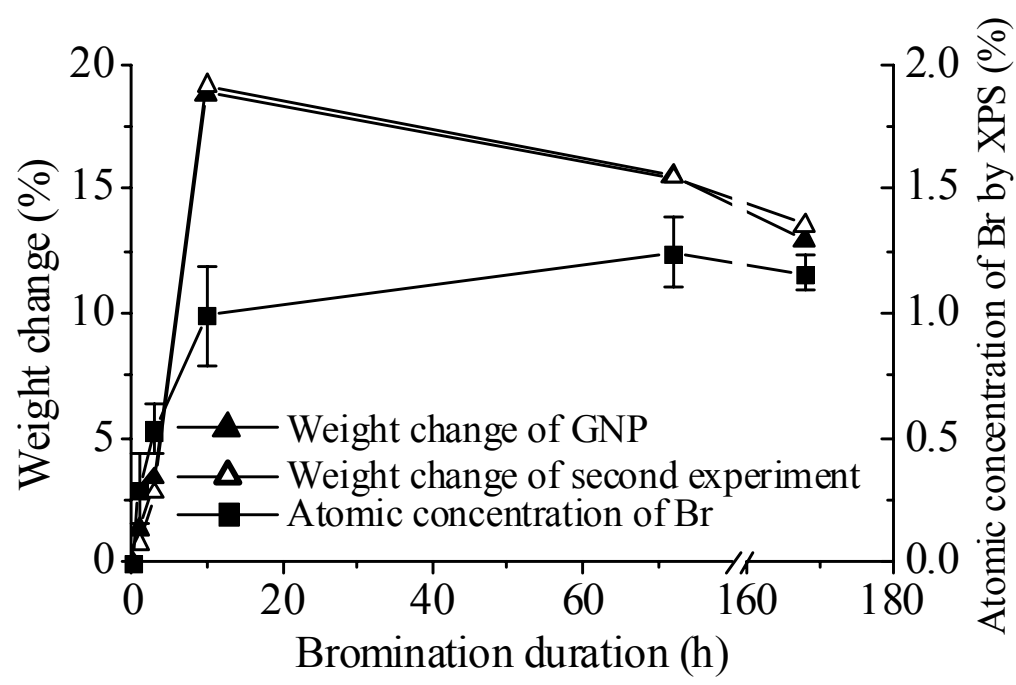

Fig. 2. Weight changes and $\mathrm{Br}$ atomic concentration determined by XPS as a function of bromination durations. 


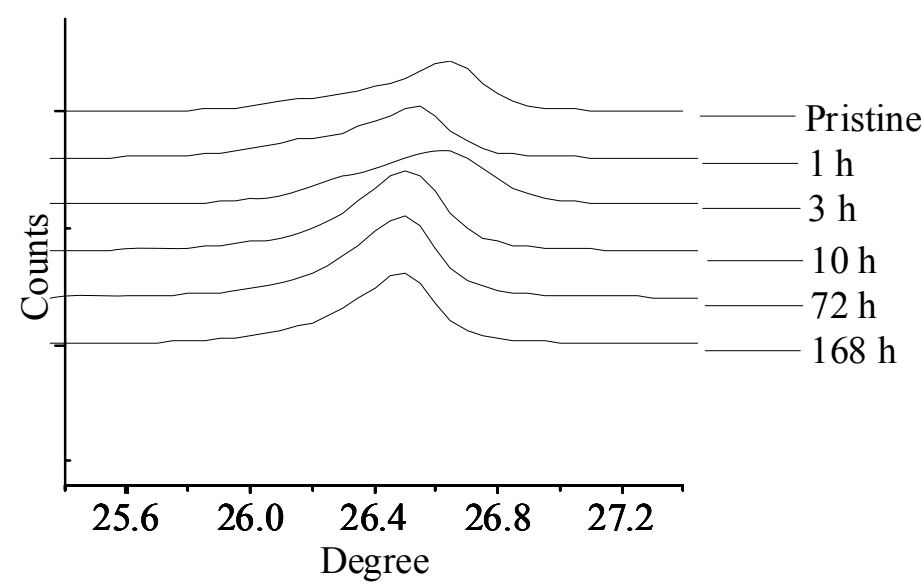

Fig.3. XRD spectra of brominated graphite with different treatment durations.

The (002) $d$-spacing of graphite is an effective quantitative measure of the extent of intercalation. The XRD spectra of brominated graphite, given in Figure 3, indicate that the peak corresponding to the $3 \mathrm{~h}$ treatment differs in position and breadth from those corresponding to the other bromination durations. Table 1 shows that with a $3 \mathrm{~h}$ treatment a point of instability is reached in which the $d$-spacing exhibits a minimum while the width-at-half-maximum (WHM) of the peak exhibits a maximum. Thereafter, for longer treatments, they stabilize on $3.361 \AA$ and $0.32^{\circ}$, respectively. The broadened peak is a reflection of the disorder of graphite crystals during the intercalation process, which is also responsible for the low $d$-spacing after the $3 \mathrm{~h}$ treatment. It appears that after a transition period, the graphite crystals become ordered again. As indicated by the WHM values, the point of maximum at the $3 \mathrm{~h}$ treatment is associated with the shortest coherence length of graphite crystals [11].

Table 1. XRD results with bromination duration

\begin{tabular}{lcccccc}
\hline Duration (h) & 0 & 1 & 3 & 10 & 72 & 168 \\
\hline d-spacing $(\AA)$ & 3.342 & 3.355 & 3.348 & 3.361 & 3.361 & 3.361
\end{tabular}




\begin{tabular}{lllllll}
$\mathrm{WHM}\left({ }^{\circ}\right)$ & 0.345 & 0.365 & 0.5 & 0.335 & 0.32 & 0.32 \\
\hline
\end{tabular}

\subsection{The nature of the $\mathrm{C}-\mathrm{Br}$ bond}

There are two possible types of $\mathrm{C}-\mathrm{Br}$ bonds in the brominated graphite, namely charge transfer complexes and covalent bonds. They are distinguishable by ToF-SIMS as shown in typical ToF-SIMS spectra of brominated GNP in Figure 4. Atomic bromine exhibits two isotopic peaks at molar masses 79 and 81 with an intensity ratio of 50.5:49.5, respectively; molecular bromine, $\mathrm{Br}_{2}$, exhibits a triplet at 158,160 and 162 with a respective intensity ratio of about 1:2:1; $\mathrm{CBr}$ exhibits a doublet at 91 and 93 ; and $\mathrm{C}_{2} \mathrm{Br}$ exhibits a doublet at 103 and 105. The reading of $\mathrm{CBr}$ and $\mathrm{C}_{2} \mathrm{Br}$ species indicates existence of covalent bond. The normalized intensities of the peaks at 91, 93, 103 and 105 are proportional to the concentration of covalently-bonded Br. The intensity was normalized by dividing the areas under the $\mathrm{CBr}$ and $\mathrm{C}_{2} \mathrm{Br}$ peaks by the area under the $\mathrm{C}_{2}$ peak (at 24), and the result is shown in Figure 5. It is seen that the normalized intensity of covalent $\mathrm{Br}$ gradually increases with increasing bromination duration.
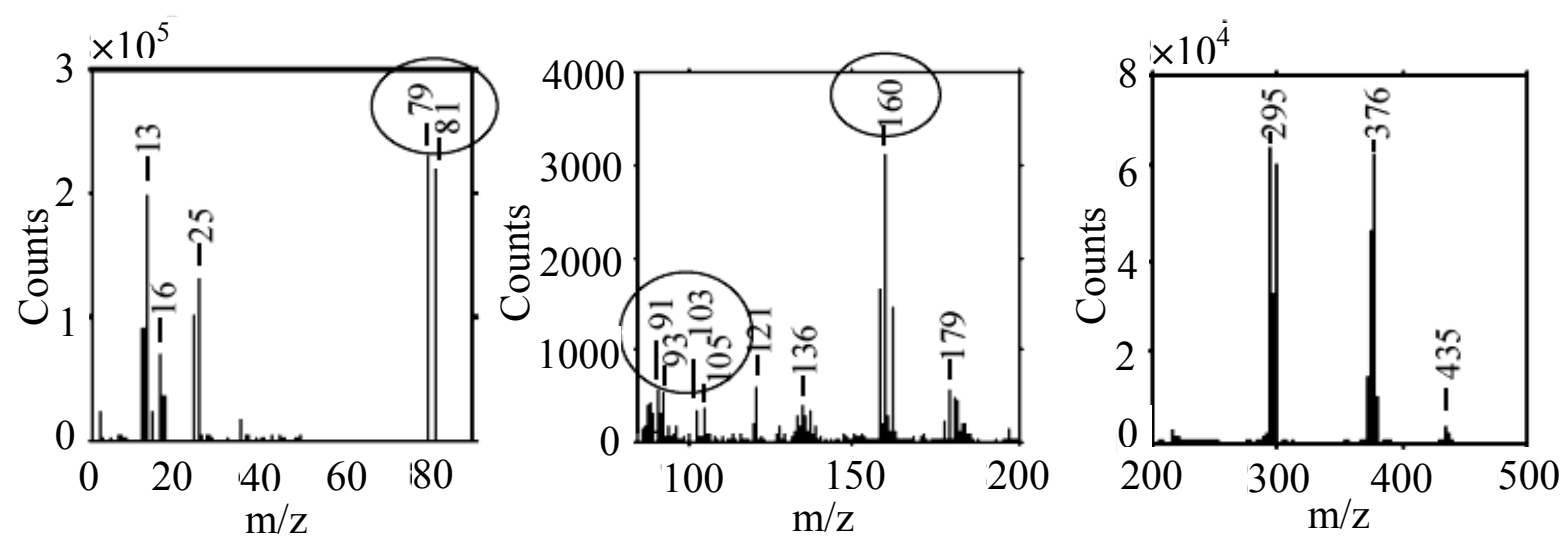

Fig. 4. ToF-SIMS spectra of brominated GNP. 


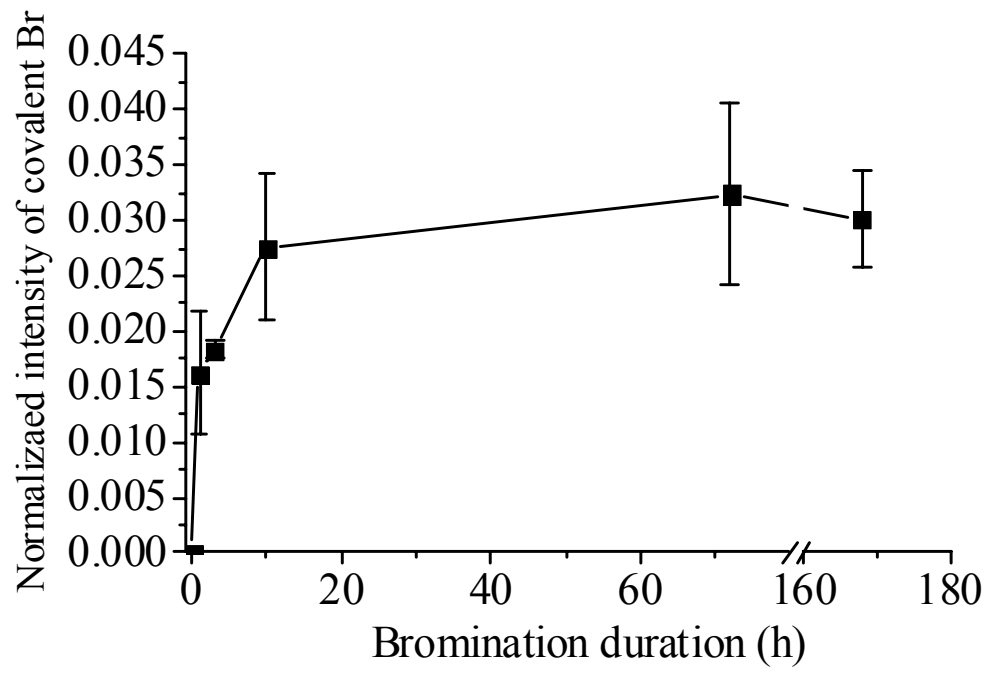

Fig. 5. Normalized intensity of covalent $\mathrm{Br}$ as a function of bromination duration.

Although the existence of various covalently bonded Br species was confirmed by the ToF-SIMS analysis, this technique could not provide quantitative information about their concentrations and relative intensities compared to ionic bonded $\mathrm{Br}$. Therefore, an XPS analysis was carried out, and typical XPS spectra corresponding to the $\mathrm{Br} 3 \mathrm{~d}$ peaks after different bromination durations are shown in Figure 6(a). The split peak originates from two different doublets superimposed in such a way that the peak located at the lower binding energy corresponds to the ionic state of $\mathrm{Br}$, while the upper one is associated with covalently bonded $\mathrm{Br}$. It is apparent that the intensities of both the total $\mathrm{Br}$ and the fraction of covalent $\mathrm{Br}$ increase with increasing the bromination duration. Quantitative analysis was performed by deconvoluting the spectrum into two doublet sets $(1+2$ and $3+4)$, as shown in Figure 6(b). In each doublet, the separation between the two peaks was $1.05 \mathrm{eV}$, the intensity ratio was 3:2 and the WHM was equal [12]. The area under the $3+4$ doublet divided by the total area under the $\mathrm{Br} 3 \mathrm{~d}$ peak was defined as the fraction of covalently bonded $\mathrm{Br}$, and the results are shown as a function of bromination duration in Figure 7. The fraction of covalent $\mathrm{Br}$ showed the 
lowest value after the $3 \mathrm{~h}$ treatment, which coincides with the highest WHM value obtained from the XRD analysis.

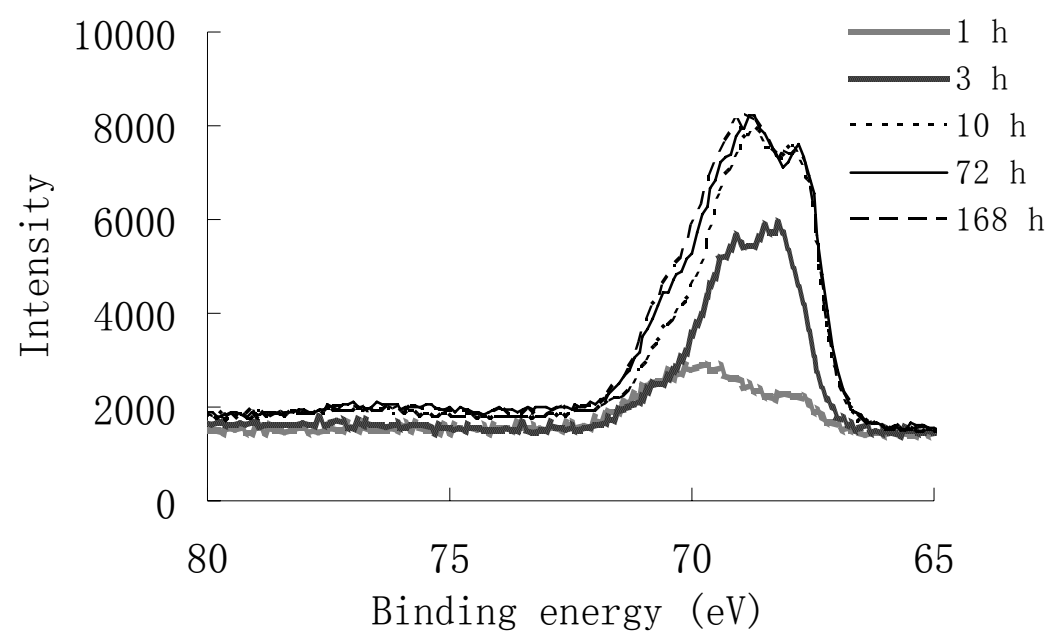

(a)

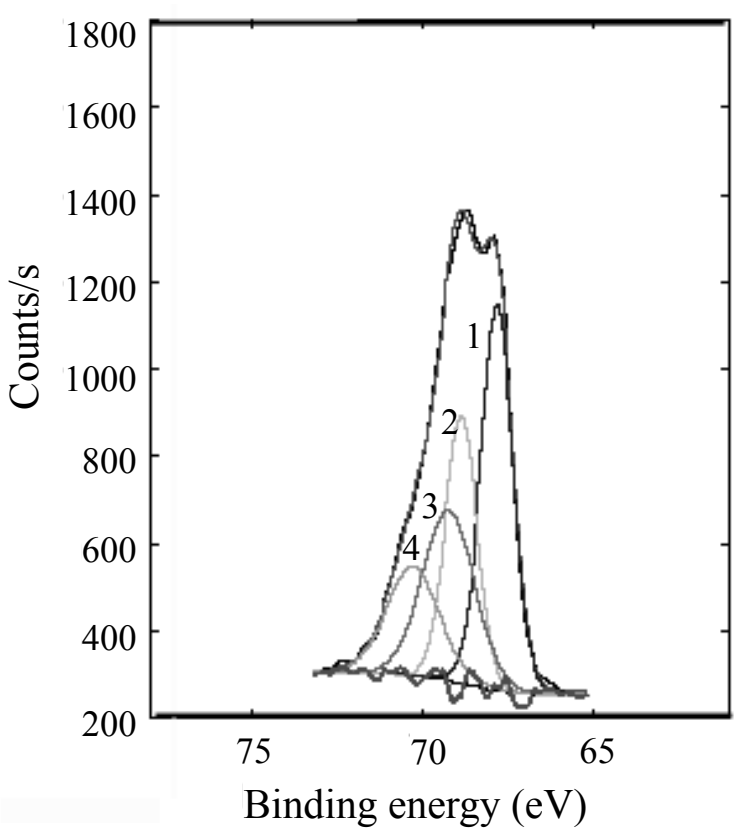

(b)

Fig. 6. XPS spectra (a) of $\mathrm{Br} 3 \mathrm{~d}$ for varying bromination durations and (b) typical deconvoluted curve fitting. 


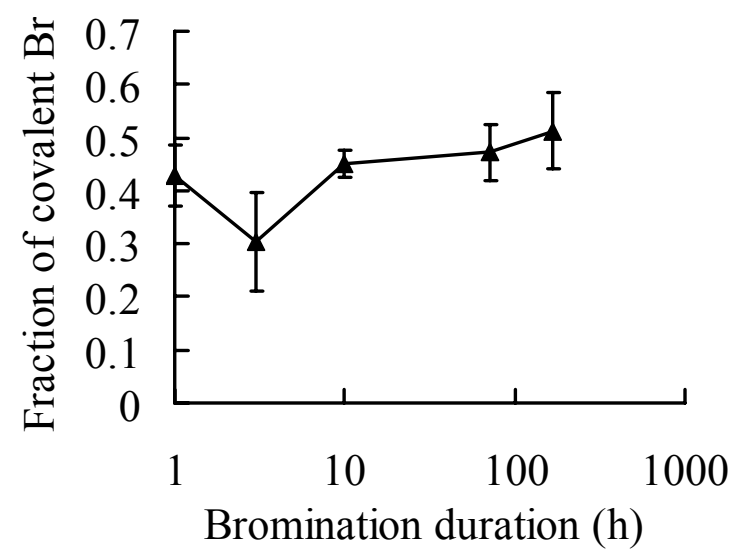

Fig. 7. Fraction of covalently bonded bromine as a function of bromination duration.

An additional quantitative study was made by plotting the atomic concentration of covalent and ionic $\mathrm{Br}$ as a function of bromination duration as shown in Figure 8 . The atomic concentrations of covalent and ionic $\mathrm{Br}$ are equivalent to the respective products of the fractions of covalent or ionic $\mathrm{Br}$ and the total atomic concentration of $\mathrm{Br}$. It is interesting to note that the atomic concentrations of both covalent and ionic $\mathrm{Br}$ increased with bromination duration and reach a plateau value after about $10 \mathrm{~h}$, indicating the saturation of bromine uptake (Figure 2). The trend of covalent $\mathrm{Br}$ with respect to bromination duration is consistent with the ToF-SIMS results shown in Figure 5. The ionic bromine concentration increased rapidly for $1 \mathrm{~h}$ to $3 \mathrm{~h}$ of bromination due to the formation of intercalation complexes between $\mathrm{Br}$ and $\mathrm{C}$, as reflected by the broadened $\mathrm{XRD}$ peak at $3 \mathrm{~h}$ of bromination (Figure 3 and Table $1)$. 


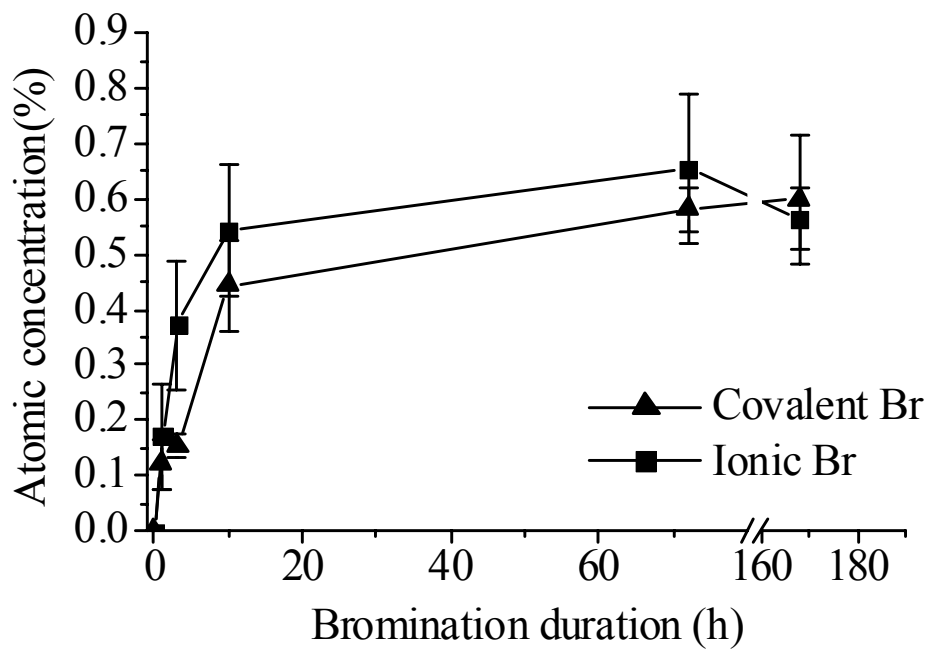

Fig. 8. Atomic concentration of covalent and ionic $\mathrm{Br}$ as a function of bromination duration.

It has been reported for carbon fibers that bromination treatment generates mainly charge transfer (ionic) bonding between $\mathrm{C}$ and $\mathrm{Br}$ and only prolonged treatment durations can generate covalent bonds [5]. Covalent $\mathrm{C}-\mathrm{Br}$ bonds can form more readily with $\mathrm{C}$ atoms at the edges of the graphitic basal plans, hence the GNP - with more edge C atoms - are more susceptible than carbon fibers to formation of covalent $\mathrm{C}-\mathrm{Br}$ bonds. So, in Figure 8, covalent and ionic Br was introduced simultaneously to GNPs. As discussed in the Introduction, ionic bonds have an ameliorating effect on the electrical conductivity of GNP because the ionic $\mathrm{Br}$ acts as an ionic acceptor. Conversely, covalent bonds decrease the electrical conductivity of GNP due to depressed carrier concentrations. As these two mechanisms cancel out each other, the final conductivity of GNP is to be determined by the ionic/covalent bond balance.

3.3 Electrical conductivity of brominated GNP and its epoxy composites

The electrical conductivity of GNP is plotted as a function of bromination duration in Figure 
9(a). After a $3 \mathrm{~h}$ treatment, the electrical conductivity rose from $353 \mathrm{~S} / \mathrm{cm}$ to reach a maximum of $540 \mathrm{~S} / \mathrm{cm}$ (an increase of $53 \%$ ), corresponding to the spike in $d$-spacing for the $3 \mathrm{~h}$ treatment (Table 1) and to the lowest fraction of covalent $\mathrm{Br}$ (Figure 7). In comparison, the electrical resistivity of different types of carbon fibers decreased by $0 \% \sim 70 \%$ after $\mathrm{Br}$ treatment [6]. The dependence of electrical conductivity of GNP is plotted as a function of the fraction of covalent $\mathrm{Br}$ in Figure 9(b). The electrical conductivity of GNP decreased with increasing the fraction of covalent $\mathrm{Br}$, which confirmed the deteriorating effect of covalent bonding on electrical conductivity as discussed above. It is interesting to note that the electrical conductivity of GNP spiked for a $3 \mathrm{~h}$ bromination treatment which coincides with an abrupt change in the $002 d$-spacing and WHM.

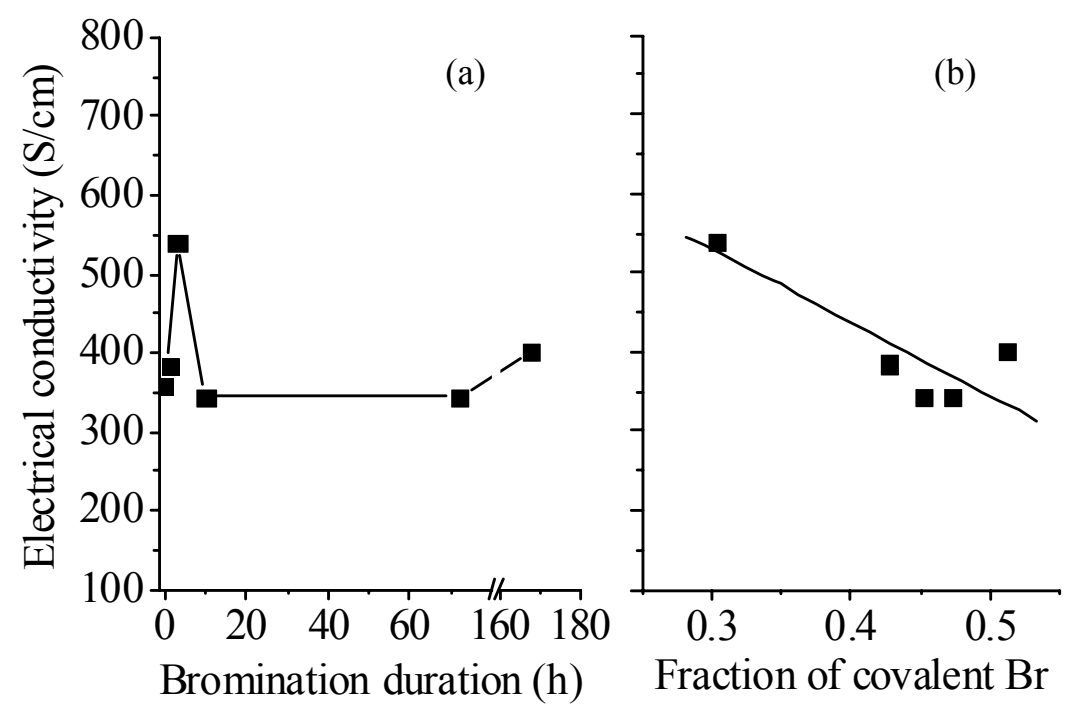

Fig. 9. Electrical conductivity of GNP as a function of (a) bromination duration; (b) fraction of covalent $\mathrm{Br}$.

Based on these observations, the $3 \mathrm{~h}$ treatment was chosen to prepare brominated GNP for GNP/epoxy composites with different GNP contents, whose electrical conductivity was 
measured. Figure 10 presents the electrical conductivity as a function of GNP content for both treated and untreated GNPs. A typical percolation phenomenon is observed with increasing GNP content: the electrical conductivity changes abruptly at around $1 \mathrm{wt} \%$ GNP, where the composite undergoes an insulator-conductor transition. To determine the percolation thresholds of the composite, the first derivatives of the conductivity plots were calculated, and the results are plotted as a function of GNP content in the inset of Figure 10. The GNP contents corresponding to the highest absolute derivatives were taken as the percolation thresholds [2], giving approximately $1 \mathrm{wt} \%$ (or approximately $0.5 \mathrm{vol} \%$ ) for the composites containing both the treated and untreated GNPs. Apparently, the percolation threshold remained unchanged after the bromination treatment because this property of conducting polymer composites depends mainly on the aspect ratio of the filler. As the bromination treatment is not expected to have a significant effect on the aspect ratio of GNP, the percolation threshold too is expected to remain unchanged.

Conversely, the bromination treatment is expected to have a significant effect on the conductivity values beyond the percolation threshold. It is seen that whereas the conductivity values of the composites below the percolation threshold are insensitive to the bromination treatment of GNP, those of the composites with more than $1 \mathrm{wt} \%$ of GNP are enhanced significantly after bromination. The maximum effect is noted for the $2.0 \mathrm{wt} \% \mathrm{GNP}$ composites for which two orders of magnitude increase is recorded for the brominated GNP composite. A combination of several mechanisms is responsible for these observations as follows. Below the percolation threshold, where GNP conducting networks in the polymer matrix do not exist yet (Figure 10), the electrical conductivity of the composite is controlled mainly by the conductivity of the matrix itself. With the formation of GNP conducting 
networks after the percolation threshold, the electrical conductivity of composites is more controlled by the corresponding properties of the filler; hence, the improved electrical conductivity of GNP affects the electrical conductivity of the composites.

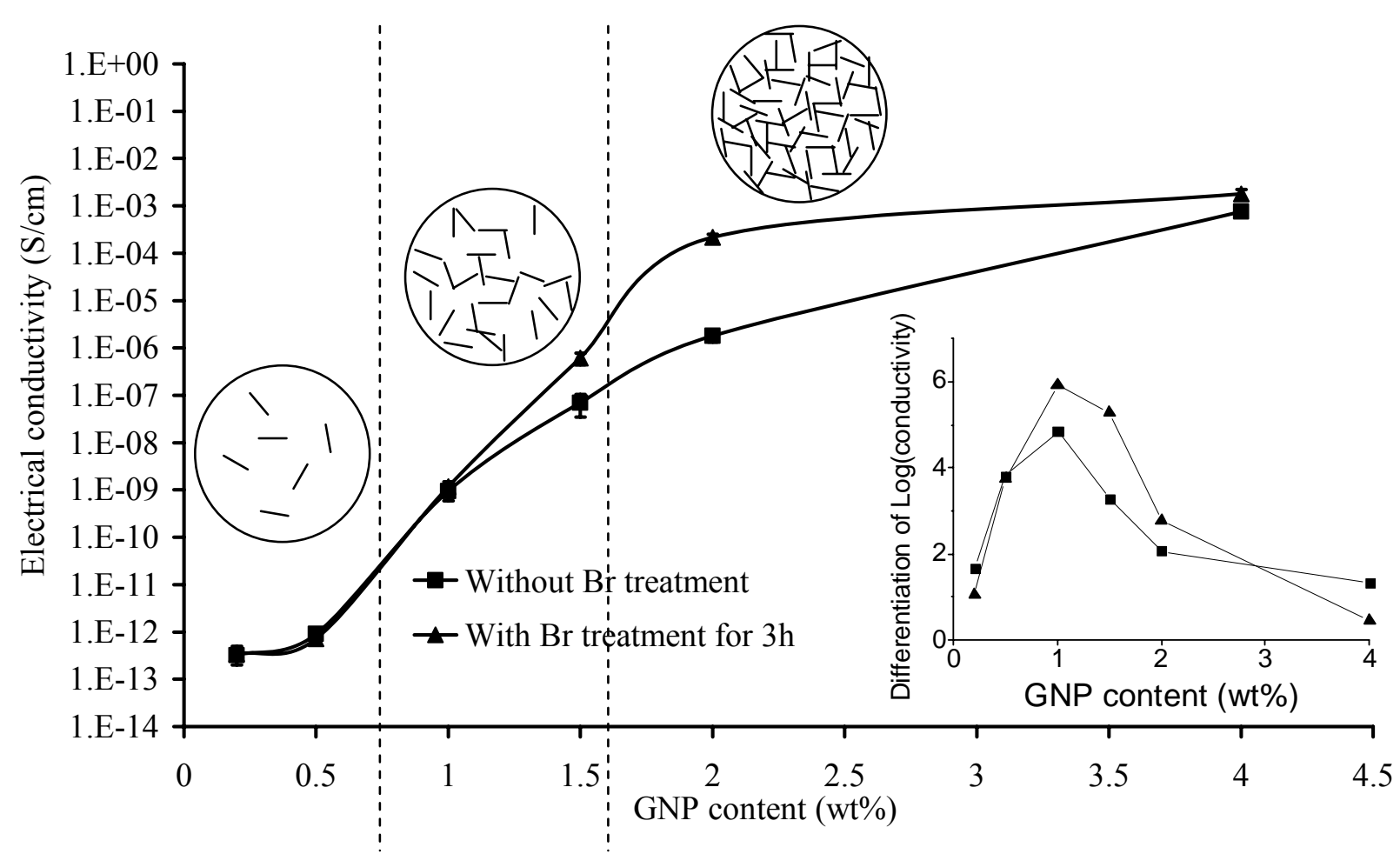

Fig. 10. Electrical conductivity of GNP/epoxy composites with and without $\mathrm{Br}$ treatment as a function of GNP content. Insertion is the absolute values of differentiation of the upper two curves.

Even beyond percolation, the conductive fillers are still wrapped by a thin layer of polymer matrix if they are well dispersed. Electrons hop between adjacent fillers by a tunneling mechanism, so that the electrical conductivity is affected also by filler-matrix interactions. The presence of covalently bonded $\mathrm{Br}$ at the edges of the graphitic basal planes 
improves the interfacial adhesion between GNP and epoxy. This is manifested by the results of the mechanical properties for treated and untreated GNPs-filled epoxy composites. Figure 11 presents the results of the flexural strength as a function of GNP content, showing significantly higher values for the brominated GNP composites. In fact, it exhibits (in the lower range of GNP content) strength values that are higher than those of the pure epoxy matrix. It is thought that the enhanced interfacial adhesion due to the presence of $\mathrm{Br}$ results also in improved electrical conductivity by eliminating unbonded gaps or micro-voids that are highly resistant to electrical conduction and are detrimental to the formation of a conductive network by GNPs. In particular, when the GNP content is close to the percolation threshold, there are only a few continuous conduction pathways throughout the bulk composite, as shown in the corresponding schematic drawing in Figure 10. Any unbonded interface or microvoids that tend to gather near the reinforcements may block the electron hopping and break up the conducting pathway, causing a significant reduction in the overall electrical conductivity. Figure 10 also suggests, however, that the electrical conductivity became rather insensitive to bromination when the GNP content was increased to $4 \mathrm{wt} \%$ GNP or above. It is envisaged that at high GNP contents far above the percolation threshold, there are many conduction pathways that crisscross the bulk composite, and the interfacial adhesion becomes rather unimportant. In this case, the difference in electrical conductivity between the composites containing GNP with and without bromination becomes negligible, only by 2.3 times in absolute terms, compared to 122 times for GNP content of $2 \mathrm{wt} \%$. 


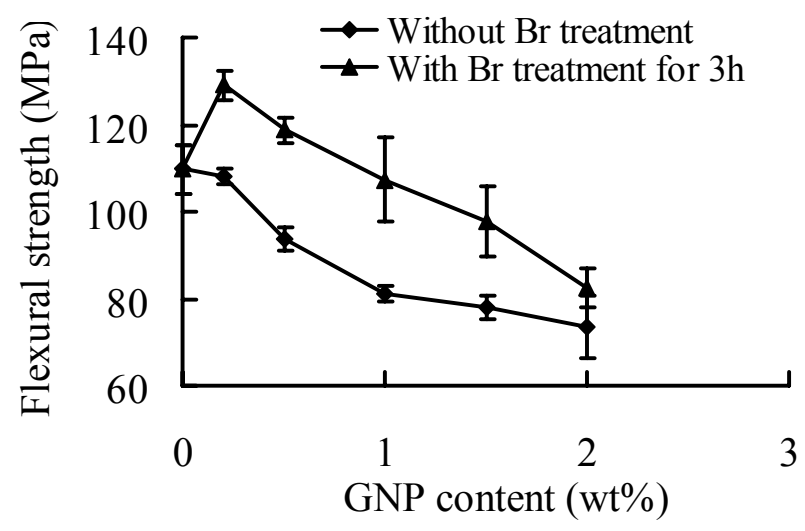

Fig. 11. Flexural strength of GNP/epoxy composites with and without Br treatment as a function of GNP content.

\section{Conclusion}

The GNPs were treated by vapor-phase bromination at room temperature for different durations. Weight increase was closely monitored, and the XPS, XRD and ToF-SIMS were employed to evaluate the intercalation of $\mathrm{Br}$ atoms and quantitatively analyze the bonding types between $\mathrm{C}$ and $\mathrm{Br}$. Electrical conductivities of the brominated GNPs and the epoxy composites were measured and compared between those with and without bromination. Major findings are highlighted as following:

1. Systematic increases in weight and atomic concentration of $\mathrm{Br}$ after bromination indicate gradual bromine uptake. The intercalation of $\mathrm{Br}$ between the graphene layers of GNP was further confirmed by the XRD result, showing an increase in d-spacing from $3.342 \AA$ to $3.361 \AA$.

2. Two types of bonds were introduced simultaneously between $\mathrm{C}$ and $\mathrm{Br}$, namely ionic and covalent bonds, both of which increased with increasing bromination duration.

3. The fraction of ionic bond reached the highest value after $3 \mathrm{~h}$ bromination, which corresponded to the highest electrical conductivity of GNP with a $52.9 \%$ improvement of 
electrical conductivity compared to the untreated GNP.

4. Bromination did not change the percolation threshold of composites. The ameliorating effect of bromination on electrical conductivity of composite became more significant when the GNP content was higher than the percolation threshold.

\section{References}

[1] Chen G, Wu C, Weng W, Wu D, Yan W. Preparation of polystyrene/graphite nanosheet composite.

Polymer 2003; 44: 1781-1784.

[2] Li J, Kim JK, Sham ML, Marom G. Morphology and properties of UV/ozone treated graphite nanoplatelet/epoxy nanocomposites.

Comp Sci Techol 2006; accepted.

[3] Jia W, Tchoudakov R, Narkis M, Siegmann A. Performance of expanded graphite and expanded milled-graphite fillers in thermosetting resins.

Polym Compos 2005; 526-533.

[4] Enoki T, Suzuki M, Endo M. Graphite intercalated compounds and applications. Oxford: Oxford University Press. 2003: 388-394.

[5] Klimenko IV, Zhuravleva TS, Geskin VM, Jawhary T. Study of the bromination of pitch-based carbon fibres.

Mater Chem Phys 1998; 56: 14-20.

[6] Mathur RB, Bahl OP, Kannan A, Flandrois A, Marchand A, Gupta V. In situ electrical resistivity changes during bromine intercalation in carbon fibres.

Carbon 1996; 34(10): 1215-1220. 
[7] Lee RS, Kim HJ, Fischer JE, Thess A, Smalley RE. Conductivity enhancement in single-walled carbon nanotube bundles doped with $\mathrm{K}$ and $\mathrm{Br}$.

Nature 1997; 388: 255-257.

[8] Prasad BLV, Sato H, Enoki T. Intercalated nanographite: structure and electronic properties.

Phys Rev B 2001; 64: 235407-1-235407-10.

[9] Li J, Kim JK, Sham ML. Conductive graphite nanoplatelet/epoxy nanocomposites: effects of exfoliation and UV/ozone treatment of graphite.

Scripta Mater 2005; 53: 235-240.

[10] Weng LT, Ng KM, Cheung ZL, Lei Y, Chan CM. Quantitative analysis of styrene-pentafluorostyrene random copolymers by ToF-SIMS and XPS.

Surf Interface Anal 2006; 38 (1): 32 - 43.

[11] Nysten B, Issi JP, Barton R, Boyington DR, Lavin JG. Determination of lattice defects in carbon fibres by means of thermal-conductivity measurements.

Phys Rev B 1991; 44(5): 2142-2148.

[12] Beamson G, Briggs D. High resolution XPS of organic polymers: the scienta ESCA300 database. Chichester, UK: Wiley. 1992: 289. 


\section{Captions for figures and tables}

Fig. 1. Process flow of GNPs and the corresponding composites.

Fig. 2. Weight changes and $\mathrm{Br}$ atomic concentration determined by XPS as a function of bromination durations.

Fig.3. XRD spectra of brominated graphite with different treatment durations.

Fig. 4. ToF-SIMS spectra of brominated GNP.

Fig. 5. Normalized intensity of covalent $\mathrm{Br}$ as a function of bromination duration.

Fig. 6. XPS spectra (a) of $\mathrm{Br} 3 \mathrm{~d}$ for varying bromination durations and (b) typical deconvoluted curve fitting.

Fig. 7. Fraction of covalently bonded bromine as a function of bromination duration.

Fig. 8. Atomic concentration of covalent and ionic $\mathrm{Br}$ as a function of bromination duration.

Fig. 9. Electrical conductivity of GNP as a function of (a) bromination duration; (b) fraction of covalent $\mathrm{Br}$.

Fig. 10. Electrical conductivity of GNP/epoxy composites with and without Br treatment as a function of GNP content. Insertion is the absolute values of differentiation of the upper two curves.

Fig. 11. Flexural strength of GNP/epoxy composites with and without $\mathrm{Br}$ treatment as a function of GNP content.

Table 1. XRD results with bromination duration. 